\title{
Orthogonal random layer lattices or random offset phase transition?
}

\author{
R. Collins*, P. Fraundorf*, D. Osborn*, and P. Sheehan*.
}

* Dept. of Physics and Astronomy and Center for Nano Science, University of Missouri - St. Louis, One University Place, St. Louis, MO, 63121.

For an infinite crystal lattice, the Laue equations predict diffraction at only specific Bragg angles. For diffraction experiments carried out on specimens that are thin in one dimension, diffraction is allowed over a range of angles that are close to the Bragg angle. In effect the reciprocal lattice points are elongated in the thin direction i.e. they appear as streaks of a length that is inversely related to the thickness of the sample. Such "rel-rod" streaks are commonly referred seen in diffraction from materials that feature random layer lattices. Example materials include Graphite and Mica. Diffraction patterns produced by such materials also exhibit unusual behavior when the specimen is tilted: Rather than disappearing under tilt, the spots in the diffraction pattern appear to move along the line of the relrod [1].

Figure 1 shows diffraction data taken from a sample of zirconium diboride known to also contain monoclinic zirconia. One can clearly see that relrods are present. Notice, however, that the relrods lie along two distinct directions and that the two directions are nearly orthogonal. It's as though the sample contains material having orthogonal random layer lattices [2]. Measurements made on the image indicate a spacing of $\mathrm{d}_{\mathrm{hkl}} \approx 5.4 \AA$ in the direction running from lower left to upper right, while the direction running from upper left to lower right has an indicated spacing of $\mathrm{d}_{\mathrm{hkl}} \approx 5.1 \AA$. The angle between the two directions is measured to be $91.3^{\circ}$. This is consistent with monoclinic zirconia being viewed down the $\mathrm{c}$ direction with $a^{*}=(100)$ and $b^{*}=(010)$.

Pure zirconia occupies the cubic phase between $\approx 2640 \mathrm{~K}$ and its melting point $\approx 2950 \mathrm{~K}$, the tetragonal phase between $\approx 1440 \mathrm{~K}$ and $\approx 2640 \mathrm{~K}$, and the monoclinic phase below $\approx 1440 \mathrm{~K}$ [3]. In cooling, zirconia transitions from a higher symmetry phase to a lower symmetry phase. During synthesis, this particular sample was raised to a temperature of $\approx 2170 \mathrm{~K}$. We expect that the zirconia present in the sample will have transitioned from the tetragonal phase to the monoclinic phase. Such phase transitions from high symmetry to low symmetry have been described by twinning [4]. Figure 2, a dark field image of the grain, clearly shows both horizontal and vertical lamellae indicating twinning. We have verified twinning by diffraction and HREM data from a similar grain. Twinning, however, is on too coarse a size scale to explain the presence of relrods.

Although an incommensurate modulation in two directions has not yet been ruled out [5], the relrods might be explained by the nucleation and growth of spatially-uncorrelated nano-precipitates during the transition from the higher-symmetry tetragonal phase. If the monoclinic precipitates are not in translational step with one another on the nano-scale, neither layering in orthogonal directions nor an incommensurate modulation may be needed to account for streaking in two or more directions [6]. 


\section{References:}

[1] Williams \& Carter, Transmission Electron Microscopy, Springer, New York, 2009.

[2] B.E. Warren, The Physical Review, Vol. 59 No. 9 (1941) 693 - 698.

[3] C.J. Howard, R.J. Hill, and B.E. Reichert, Acta Cryst., B44 (1988) 116 - 120.

[4] J. E. Bailey (1964) Proc.Roy. Soc 279 p395; N.K. Simha (1997) J.Mech.Phys.Solids 45 p261.

[5] cf. Joke Hadermann (2011) video tutorial at http://www.ustream.tv/recorded/15283038.

[6] This research was carried out with the support of Air Force contract number FA8650-05-D-5807. The author wishes to acknowledge David Osborn for preparing the sample.

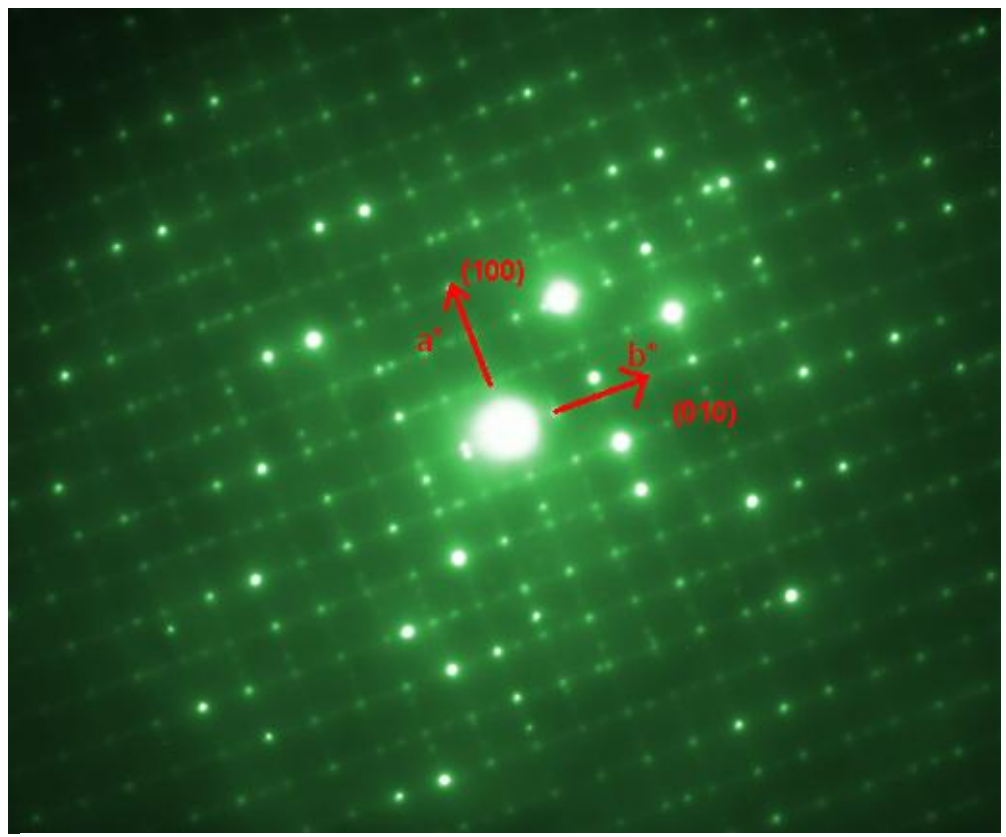

Figure 1: Diffraction data from the zirconia grain showing relrods present along the $a^{*}$ and $b^{*}$ directions.

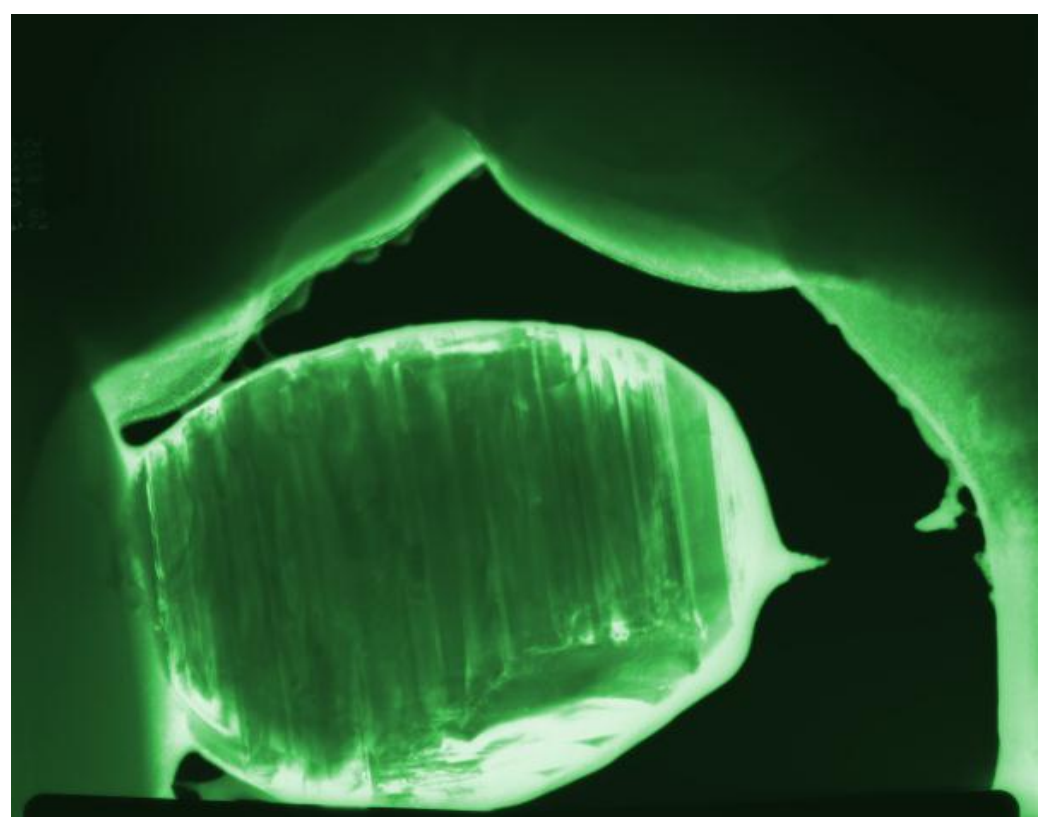

Figure 3: A darkfield image of the zirconia grain showing vertical and horizontal lamellae.
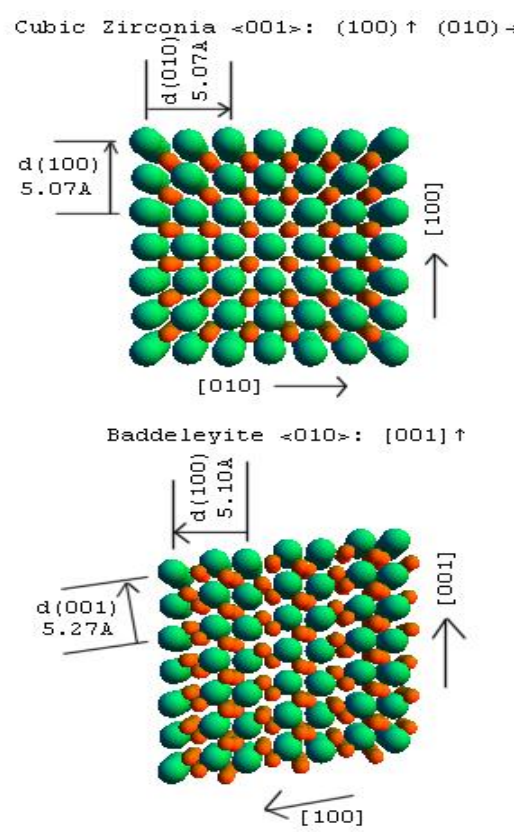

Figure 2: $\mathrm{c}^{-\mathrm{ZrO}_{2}}$ and $\mathrm{m}-\mathrm{ZrO}_{2}$ 